\title{
Circular Chromatic Ramsey Number
}

\author{
Kyle F. Jao*, Claude Tardif ${ }^{\dagger}$ Douglas B. West ${ }^{\ddagger}$ Xuding Zhu ${ }^{\S}$
}

Revised May 2014

\begin{abstract}
Let $\chi_{c}(H)$ denote the circular chromatic number of a graph $H$. For graphs $F$ and $G$, the circular chromatic Ramsey number $R_{\chi_{c}}(F, G)$ is the infimum of $\chi_{c}(H)$ over graphs $H$ such that every red/blue edge-coloring of $H$ contains a red copy of $F$ or a blue copy of $G$. We characterize $R_{\chi_{c}}(F, G)$ in terms of a Ramsey problem for the families of homomorphic images of $F$ and $G$. Letting $z_{k}=3-2^{-k}$, we prove that $z_{k-1} \leq \chi_{c}(G) \leq z_{k}$ implies $2 z_{k-1} \leq R_{\chi_{c}}(G, G) \leq 2 z_{k}$. For integer $k$ with $k>1$, there is a graph $G$ with $\chi_{c}(G) \geq k$ and $R_{\chi_{c}}(G, G) \leq k(k-1)$. Our most difficult result is $R_{\chi_{c}}(F, G)=4$ when $\chi_{c}(F), \chi_{c}(G) \in\left(2, \frac{5}{2}\right]$. As a consequence, no graph $G$ satisfies $4<R_{\chi_{c}}(G, G)<5$. We also prove $\frac{14}{3} \leq R_{\chi_{c}}\left(C_{3}, C_{5}\right) \leq 5$ and $4 \leq R_{\chi_{c}}\left(C_{3}, C_{7}\right) \leq \frac{9}{2}$.
\end{abstract}

\section{Introduction}

Given families $\mathcal{F}$ and $\mathcal{G}$ of graphs, classical graph Ramsey theory considers graphs $H$ such that every red/blue edge-coloring of $H$ contains a red graph from $\mathcal{F}$ or a blue graph from $\mathcal{G}$; we then write $H \rightarrow(\mathcal{F}, \mathcal{G})$ and say that $H$ arrows $(\mathcal{F}, \mathcal{G})$. The Ramsey number $R(\mathcal{F}, \mathcal{G})$ is $\min \{|V(H)|: H \rightarrow(\mathcal{F}, \mathcal{G})\}$. (When $\mathcal{F}$ or $\mathcal{G}$ is a single graph, we drop set braces.)

For any mononotone graph parameter $\rho$, the $\rho$-Ramsey number of $(\mathcal{F}, \mathcal{G})$, written $R_{\rho}(\mathcal{F}, \mathcal{G})$, is $\inf \{\rho(H): H \rightarrow(\mathcal{F}, \mathcal{G})\}$. Besides the number of vertices, the notion has been studied with $\rho$ being the clique number $[4,10]$, chromatic number $[2,14]$, number of edges (yielding the "size Ramsey number") $[3,11]$, and maximum degree $[8,9]$. In this paper we let $\rho$ be the "circular chromatic number".

The circular chromatic number of a graph $G$, written $\chi_{c}(G)$, is the infimum of $p / q$ such that the vertices of $H$ can be assigned congruence classes modulo $p$ so that the classes of adjacent vertices differ by at least $q$. Formally, for $p, q \in \mathbb{N}$ with $p \geq 2 q$, the generalized

\footnotetext{
${ }^{*}$ Department of Mathematics, University of Illinois, Urbana, IL, fjao2@illinois.edu

†Department of Mathematics and Computer Science, Royal Military College of Canada, Kingston, ON, Canada, Claude.Tardif@rmc.ca

${ }^{\ddagger}$ Department of Mathematics, Zhejiang Normal University, Jinhua, China, and University of Illinois, Urbana, IL, west@math.uiuc.edu; research supported in part by NSA grant H98230-10-1-0363.

${ }^{\S}$ Department of Mathematics, Zhejiang Normal University, Jinhua, China, xudingzhu@gmail.com; research supported in part by NSFC No. 11171730 and ZJNSF No. Z6110786.
} 
complete graph or circular clique $K_{p: q}$ has vertex set $\left\{v_{i}: 0 \leq i \leq p-1\right\}$ and edge set $\left\{v_{i} v_{j}: q \leq|i-j| \leq p-q\right\}$. A homomorphism from $G$ to $H$ is a map $\phi: V(G) \rightarrow V(H)$ that preserves edges. When a homomorphism exists from $G$ to $K_{p: q}$, we say that $G$ is $(p, q)$ colorable, so $\chi_{c}(G)=\inf \{p / q: G$ is $(p, q)$-colorable $\}$. If $G$ is finite, then $\chi_{c}(G)$ is rational and the infimum is the minimum $[1,13]$.

Since a $(p, 1)$-coloring is just an ordinary proper $p$-coloring, $\chi_{c}(G) \leq \chi(G)$. It is also well known that always $\chi_{c}(G)>\chi(G)-1[1,13]$. Hence in fact $\chi(G)=\left\lceil\chi_{c}(G)\right\rceil$, meaning that the circular chromatic number is a refinement of the chromatic number.

Letting $\rho=\chi_{c}$, we study the circular chromatic Ramsey number:

$$
R_{\chi_{c}}(\mathcal{F}, \mathcal{G})=\inf \left\{\chi_{c}(H): H \rightarrow(\mathcal{F}, \mathcal{G})\right\} .
$$

Here the infimum is needed; the minimum may not exist.

Circular chromatic Ramsey number also arises from a different extension of Ramsey number. In studying $R(\mathcal{F}, \mathcal{G})$, we seek monochromatic isomorphic images of target graphs. For $K_{n}$ we could equivalently seek homomorphic images. If we seek monochromatic homomorphic images in general, then the minimum number of vertices in a complete graph that suffices is $R_{\chi}(\mathcal{F}, \mathcal{G})$, by a result of [2] that we will state later. If we extend the options for the host to all $K_{p: q}$, then the resulting parameter is equal to $R_{\chi_{c}}(\mathcal{F}, \mathcal{G})$.

In Section 2, we discuss the basic properties of $R_{\chi_{c}}$ and characterize $R_{\chi_{c}}(F, G)$ in terms of which circular cliques arrow the families of homomorphic images of $F$ and $G$. Section 3 begins with easy arguments for small graphs analogous to the simplest Ramsey problems, obtaining $R_{\chi_{c}}\left(K_{3}, K_{n}\right)=R\left(K_{3}, K_{n}\right)$ for $n \in\{3,4,5\}$. However, $R_{\chi_{c}}\left(K_{4}, K_{4}\right) \leq 17.5<18=$ $R\left(K_{4}, K_{4}\right)$. We then obtain bounds on $R_{\chi_{c}}(G)$ whenever $2 \leq \chi_{c}(G) \leq 3$. Letting $z_{t}=3-2^{-t}$, we show that $z_{t-1} \leq \chi_{c}(G) \leq z_{t}$ implies $2 z_{t-1} \leq \chi_{c}(G) \leq 2 z_{t}$. This yields non-integer values of $R_{\chi_{c}}$ between 5 and 6 when $t \geq 2$.

For integer $k$ with $k>2$, in Section 4 we show the existence of a graph $G_{k}$ with $\chi_{c}\left(G_{k}\right) \geq$ $k$ and $R_{\chi_{c}}\left(G_{k}\right) \leq k(k-1)$. This supports an analogue of the conjecture from [2] that $R_{\chi}(k)=(k-1)^{2}+1$, where $R_{\chi}(k)=\inf \left\{R_{\chi}(G, G): \chi(G)=k\right\}$, which was proved by Zhu [15]. This result shows that the values of $R_{\chi_{c}}(G, G)$ vary exponentially when $\chi_{c}(G)$ is fixed, since $R_{\chi_{c}}\left(K_{k}, K_{k}\right) \geq R_{\chi}\left(K_{k}, K_{k}\right)-1=R(k, k)-1>O\left(k 2^{k / 2}\right)$, using the well-known lower bound on classical Ramsey numbers.

Returning to smaller values, in Section 5 we prove our most difficult result: $R_{\chi_{c}}(G)=4$ whenever $2 \leq \chi_{c}(G) \leq 5 / 2$. Combined with the observations of Section 2, we conclude that if each of $\mathcal{F}$ and $\mathcal{G}$ contains no bipartite graph but contains a graph with circular chromatic number at most $5 / 2$ (such as an odd cycle of length at least 5 ), then $R_{\chi_{c}}(\mathcal{F}, \mathcal{G})=4$. Furthermore, there is no graph $G$ such that $4<R_{\chi_{c}}(G, G)<5$.

For the "non-diagonal" case, In Section 6 we prove $\frac{14}{3} \leq R_{\chi_{c}}\left(C_{3}, C_{5}\right) \leq 5$ and $4 \leq$ $R_{\chi_{c}}\left(C_{3}, C_{7}\right) \leq \frac{9}{2}$. In contrast to $R_{\chi_{c}}\left(C_{2 k+1}, C_{2 k+1}\right)=4$ for $k \geq 2$, this implies $R_{\chi_{c}}\left(C_{3}, C_{5}\right) \neq$ $R_{\chi_{c}}\left(C_{3}, C_{7}\right)$, though we do not yet know either value. 


\section{General Observations}

The classical bounds $\chi(H)-1<\chi_{c}(H) \leq \chi(H)$ yield analogous bounds on $R_{\chi_{c}}(\mathcal{F}, \mathcal{G})$.

Proposition 2.1. $R_{\chi}(\mathcal{F}, \mathcal{G})-1 \leq R_{\chi_{c}}(\mathcal{F}, \mathcal{G}) \leq R_{\chi}(\mathcal{F}, \mathcal{G})$.

Proof. Since $\chi_{c}(H) \leq \chi(H)$ when $H \rightarrow(\mathcal{F}, \mathcal{G})$, we have $R_{\chi_{c}}(\mathcal{F}, \mathcal{G}) \leq R_{\chi}(\mathcal{F}, \mathcal{G})$. For the other inequality, if $k \leq R_{\chi_{c}}(\mathcal{F}, \mathcal{G})<k+1$ for some integer $k$, then $H \rightarrow(\mathcal{F}, \mathcal{G})$ for some graph $H$ with $k \leq \chi_{c}(H)<k+1$. Since $k \leq \chi(H) \leq k+1$, we have $R_{\chi}(\mathcal{F}, \mathcal{G}) \leq k+1$, and hence $R_{\chi}(\mathcal{F}, \mathcal{G}) \leq R_{\chi_{c}}(\mathcal{F}, \mathcal{G})+1$.

Early work on the circular chromatic number asked when $\chi_{c}(H)$ equals $\chi(H)$ or is close to $\chi(H)-1$. We ask the same question for the bounds on $R_{\chi_{c}}(\mathcal{F}, \mathcal{G})$ in Proposition 2.1.

Like the chromatic Ramsey number in [2], the circular chromatic Ramsey number can be phrased as a classical graph Ramsey problem using homomorphisms. If there is a homomorphism from $H$ to $H^{\prime}$, then $H^{\prime}$ need not contain every subgraph in $H$, but it contains a homomorphic image of every subgraph in $H$. The resulting lemma will help us rephrase $R_{\chi_{c}}$ using circular cliques. Given a family $\mathcal{F}$, let $\operatorname{Hom}(\mathcal{F})$ denote the family of all homomorphic images of graphs in $\mathcal{F}$ via surjective maps.

Lemma 2.2. If $H \rightarrow(\mathcal{F}, \mathcal{G})$ and there is a homomorphism $\phi: H \rightarrow H^{\prime}$, then $H^{\prime} \rightarrow(\operatorname{Hom}(\mathcal{F}), \operatorname{Hom}(\mathcal{G}))$.

Proof. Let $f^{\prime}$ be a red/blue edge-coloring of $H^{\prime}$. For $e \in E(H)$, let $f(e)=f^{\prime}(\phi(e))$. Under $f$, in $H$ there is a red subgraph belonging to $\mathcal{F}$ or a blue subgraph belonging to $\mathcal{G}$. This subgraph maps under $\phi$ to a subgraph of $H$ in $\operatorname{Hom}(\mathcal{F})$ or $\operatorname{Hom}(\mathcal{G})$ that has the specified color under $f^{\prime}$. Hence $H^{\prime} \rightarrow(\operatorname{Hom}(\mathcal{F}), \operatorname{Hom}(\mathcal{G}))$.

Burr, Erdős, and Lovász [2] proved

$$
R_{\chi}(\mathcal{F}, \mathcal{G})=R(\operatorname{Hom}(\mathcal{F}), \operatorname{Hom}(\mathcal{G}))=\inf \left\{n: K_{n} \rightarrow(\operatorname{Hom}(\mathcal{F}), \operatorname{Hom}(\mathcal{G}))\right\}
$$

They used the bipartite version of Ramsey's Theorem and the blowup $J[n]$ of a graph $J$, defined to be the graph obtained from $J$ by expanding each vertex into an independent set of size $n$ (and each edge into a copy of the complete bipartite graph $K_{n, n}$ ). Their idea applies also to $R_{\chi_{c}}$, yielding the following result.

Theorem 2.3. If $\mathcal{F}$ and $\mathcal{G}$ are finite, then $R_{\chi_{c}}(\mathcal{F}, \mathcal{G})=\inf \left\{p / q: K_{p: q} \rightarrow(\operatorname{Hom}(\mathcal{F}), \operatorname{Hom}(\mathcal{G}))\right\}$.

Proof. Whenever $H \rightarrow(\mathcal{F}, \mathcal{G})$ with $\chi_{c}(H)=p / q$, Lemma 2.2 yields $K_{p: q} \rightarrow(\operatorname{Hom}(\mathcal{F}), \operatorname{Hom}(\mathcal{G}))$. Thus $\inf \left\{p / q: K_{p: q} \rightarrow(\operatorname{Hom}(\mathcal{F}), \operatorname{Hom}(\mathcal{G}))\right\} \leq R_{\chi_{c}}(\mathcal{F}, \mathcal{G})$.

For the reverse inequality, suppose $K_{p: q} \rightarrow(\operatorname{Hom}(\mathcal{F}), \operatorname{Hom}(\mathcal{G}))$. Let $H=K_{p: q}[n]$, where $n$ is the largest number of vertices among graphs in $\mathcal{F}$ or $\mathcal{G}$. When $N$ is sufficiently large, 
every 2-edge-coloring of $H[N]$ contains a copy of $H$ in which each copy of $K_{n, n}$ corresponding to a single edge of $K_{p: q}$ is monochromatic. This is proved by iterating the bipartite version of Ramsey's Theorem, as in [2]. The resulting 2-edge-coloring of $K_{p: q}$ has a red copy of some graph in $\operatorname{Hom}(\mathcal{F})$ or a blue copy of some graph in $\operatorname{Hom}(\mathcal{G})$, since $K_{p: q} \rightarrow(\operatorname{Hom}(\mathcal{F}), \operatorname{Hom}(\mathcal{G}))$. In the 2-edge-coloring of $H$, this subgraph expands into a monochromatic copy of the corresponding graph in $\mathcal{F}$ or $\mathcal{G}$ with the right color. Hence $H[N] \rightarrow(\mathcal{F}, \mathcal{G})$. Since $\chi_{c}(H[N])=p / q$, we have $R_{\chi_{c}}(\mathcal{F}, \mathcal{G}) \leq \inf \left\{p / q: K_{p: q} \rightarrow(\operatorname{Hom}(\mathcal{F}), \operatorname{Hom}(\mathcal{G}))\right\}$.

Lemma 2.4. If each graph in the family $\mathcal{F}^{\prime}$ contains a homomorphic image of some graph in the family $\mathcal{F}$, and similarly for $\mathcal{G}^{\prime}$ and $\mathcal{G}$, then $R_{\chi_{c}}(\mathcal{F}, \mathcal{G}) \leq R_{\chi_{c}}\left(\mathcal{F}^{\prime}, \mathcal{G}^{\prime}\right)$.

Proof. By hypothesis, $\operatorname{Hom}\left(\mathcal{F}^{\prime}\right) \subseteq \operatorname{Hom}(\mathcal{F})$ and $\operatorname{Hom}\left(\mathcal{G}^{\prime}\right) \subseteq \operatorname{Hom}(\mathcal{G})$. Therefore, $K_{p: q} \rightarrow$ $\left(\operatorname{Hom}\left(\mathcal{F}^{\prime}\right), \operatorname{Hom}\left(\mathcal{G}^{\prime}\right)\right)$ implies $K_{p: q} \rightarrow(\operatorname{Hom}(\mathcal{F}), \operatorname{Hom}(\mathcal{G}))$. Hence

$$
\begin{aligned}
R_{\chi_{c}}(\mathcal{F}, \mathcal{G}) & =\inf \left\{p / q: K_{p: q} \rightarrow(\operatorname{Hom}(\mathcal{F}), \operatorname{Hom}(\mathcal{G}))\right\} \\
& \leq \inf \left\{p / q: K_{p: q} \rightarrow\left(\operatorname{Hom}\left(\mathcal{F}^{\prime}\right), \operatorname{Hom}\left(\mathcal{G}^{\prime}\right)\right)\right\}=R_{\chi_{c}}\left(\mathcal{F}^{\prime}, \mathcal{G}^{\prime}\right)
\end{aligned}
$$

When $\mathcal{F}=\mathcal{G}=\{G\}$, we write $H \rightarrow G$ for $H \rightarrow(G, G)$ and abbreviate the argument to the various Ramsey numbers as $G$. Lemma 2.4 yields $R_{\chi_{c}}(G) \leq R_{\chi_{c}}\left(K_{p: q}\right)$ when $\chi_{c}(G)=p / q$, so we study $R_{\chi_{c}}\left(K_{p: q}\right)$ to obtain upper bounds on $R_{\chi_{c}}(G)$, especially when $\chi_{c}(G)$ is small. In Section 3, we compute circular chromatic Ramsey numbers for small complete graphs and obtain bounds for some circular cliques. Since $C_{2 k+1} \cong K_{(2 k+1): 2}$, this theme continues when we consider odd cycles and pairs of odd cycles in Sections 5 and 6 .

Theorem 5.4 proves $R_{\chi_{c}}\left(C_{5}\right)=4$. Using the general observations above, this result implies the following.

Corollary 2.5. $R_{\chi_{c}}(\mathcal{F}, \mathcal{G}) \leq 4$ whenever $\mathcal{F}$ and $\mathcal{G}$ each contain some graph with circular chromatic number at most $\frac{5}{2}$. Equality holds when neither $\mathcal{F}$ nor $\mathcal{G}$ contains a bipartite graph.

Proof. Every graph with circular chromatic number at most 5/2 admits a homomorphism into $C_{5}$. Thus $\mathcal{F}^{\prime}=\mathcal{G}^{\prime}=\left\{C_{5}\right\}$ in Lemma 2.4 yields the upper bound. For the lower bound, every graph with chromatic number at most 4 decomposes into two bipartite graphs.

From the characterization of $R_{\chi}(G)$ in [2], it follows that $R_{\chi}(G) \in\{5,6\}$ when $G$ is 3 -chromatic, and the value is 5 if and only if $G$ is $(5,2)$-colorable. On the other hand, $2<\chi_{c}(G) \leq \frac{5}{2}$ implies $R_{\chi_{c}}(G)=4$. When $\frac{5}{2}<\chi_{c}(G) \leq 3$, we have $R_{\chi}(G)=6$. Since Proposition 2.1 yields $R_{\chi}(G)-1 \leq R_{\chi_{c}}(G) \leq R_{\chi}(G)$, it follows that $\frac{5}{2}<\chi_{c}(G) \leq 3$ implies $5 \leq R_{\chi_{c}}(G) \leq 6$, and hence there are no circular chromatic Ramsey numbers between 4 and 5. However, we show in Section 3 that there are such values between 5 and 6 , using the following remark. 
Remark 2.6. Upper and lower bounds. By Theorem $2.3, K_{p: q} \rightarrow \operatorname{Hom}(G)$ implies $R_{\chi_{c}}(G) \leq$ $p / q$. Also, if $K_{p: q} \rightarrow \operatorname{Hom}(G)$ and $p^{\prime} / q^{\prime} \geq p / q$, then $K_{p^{\prime}: q^{\prime}} \rightarrow \operatorname{Hom}(G)$, by Lemma 2.2. The homomorphic images of $G$ all have circular chromatic number at least $\chi_{c}(G)$ and include all $K_{r: s}$ with $r / s \geq \chi_{c}(G)$. Therefore, if there exists $K_{r: s}$ having a red/blue edge-coloring such that each color class has circular chromatic number less than $\chi_{c}(G)$, then $R_{\chi_{c}}(G) \geq r / s$. We use this method in Section 3 to obtain upper and lower bounds showing that if $\frac{11}{4} \leq$ $\chi_{c}(G)<3$, then $5.5<R_{\chi_{c}}(G)<6$.

\section{Bounds and Small Values}

Using the known colorings that establish lower bounds for $R\left(K_{3}, G\right)$, we prove $R_{\chi_{c}}\left(K_{3}, G\right)=$ $R\left(K_{3}, G\right)$ for $G \in\left\{K_{3}, K_{4}, K_{5}\right\}$. Since always $R_{\chi_{c}}(F, G) \leq R(F, G)$, we need only discuss the lower bounds. Let an $(\mathcal{F}, \mathcal{G})$-avoidance on $H$ be a red/blue edge-coloring of $H$ having no red subgraph in $\mathcal{F}$ and no blue subgraph in $\mathcal{G}$.

The length of an edge $v_{i} v_{j}$ in $K_{p: q}$ is $\min \{|i-j|, p-|i-j|\}$. Recall that $\operatorname{Hom}\left(K_{n}\right)=\left\{K_{n}\right\}$.

Proposition 3.1. $R_{\chi_{c}}\left(K_{3}, K_{3}\right)=R\left(K_{3}, K_{3}\right)=6$.

Proof. It suffices to find for all $q$ a $\left(K_{3}, K_{3}\right)$-avoidance on $K_{(6 q-1): q}$. Assign red to the edges with lengths $q, \ldots, 2 q-1$, and blue to the edges with lengths $2 q, \ldots,\left\lfloor\frac{(6 q-1)}{2}\right\rfloor$.

The lengths of the edges of a triangle in $K_{(6 q-1): q}$ are $a, b, c$ with $a+b+c=6 q-1$ or $a+b=c$. For red edges of any lengths $a, b, c$, we have $a+b+c \leq 6 q-3<6 q-1$ and $a+b \geq 2 q>c$. For blue edges of these lengths, we have $a+b+c \geq 6 q>6 q-1$ and $a+b \geq 4 q>c$. With a contradiction in each case, there is no monochromatic triangle.

Proposition 3.2. $R_{\chi_{c}}\left(K_{3}, K_{4}\right)=R\left(K_{3}, K_{4}\right)=9$.

Proof. It suffices to find for all $q$ a $\left(K_{3}, K_{4}\right)$-avoidance on $K_{(9 q-1): q}$. Assign blue to all edges with lengths $q, \ldots, 3 q-1$ and red to the edges with lengths $3 q, \ldots,\left\lfloor\frac{(9 q-1)}{2}\right\rfloor$.

The lengths of the three edges of a triangle in $K_{(9 q-1): q}$ are $a, b, c$ with $a \leq b \leq c$ so that $a+b+c=9 q-1$ or $a+b=c \leq(9 q-1) / 2$. Since the lengths of three red edges sum to at least $9 q$, and two sum to at least $6 q$, there is no red triangle.

Consider the four vertices of a 4-clique in $K_{(9 q-1): q}$ in cyclic order of indices. The edge joining two opposite vertices $u$ and $v$ forms triangles with each of the two remaining vertices, $x$ and $y$. In one of these triangles, the three lengths sum to $9 q-1$. Since three blue lengths sum to at most $9 q-3$, there is no blue copy of $K_{4}$.

The particular coloring in Proposition 3.2 was noticed by Daniel Cranston. It is slightly simpler than our original coloring.

Proposition 3.3. $R_{\chi_{c}}\left(K_{3}, K_{5}\right)=R\left(K_{3}, K_{5}\right)=14$. 
Proof. It suffices to find for all $q$ a $\left(K_{3}, K_{5}\right)$-avoidance on $K_{(14 q-1): q}$. Assign red to all edges with lengths $2 q, \ldots, 4 q-1$ and blue to all other edges.

Since $12 q-3<14 q-1$, a red triangle can occur only by using two red lengths that sum to a red length, but $2 q+2 q>4 q-1$ prevents this.

It remains to prohibit blue 5-cliques. Blue edges include short edges with lengths from $q$ to $2 q-1$ and long edges with lengths at least $4 q$. Consider the separations among consecutive vertices of a blue 5 -clique when viewed in cyclic order of subscripts. The separations must sum to $14 q-1$ using blue lengths. The sum of two short blue lengths is between $2 q$ and $4 q-2$, all of which are red lengths, so a blue 5-clique cannot have consecutive short separations. Hence it has at most two short separations. Since the long separations are at least $4 q$ each, the sum of the five separations in a blue 5-clique is at least $14 q$, a contradiction.

The behavior of $R_{\chi_{c}}\left(K_{4}, K_{4}\right)$ is quite different. Although $R\left(K_{4}, K_{4}\right)=18$, we will prove $R_{\chi_{c}}\left(K_{4}, K_{4}\right) \leq 17.5$. Greenwood and Gleason [6] showed $R\left(K_{4}, K_{4}\right)=18$ by coloring $K_{17}$ with no monochromatic 4-clique, using cyclic symmetry. Viewed as $K_{17: 1}$, the edges of lengths 1,2,4,8 are one color, and those of lengths 2, 5, 6,7 are the other color. The coloring has monochromatic 4-cycles, but their chords do not both have that color. In fact, in every $\left(K_{4}, K_{4}\right)$-avoidance on $K_{17}$, each color class is a regular subgraph. The next lemma will enable us to take advantage of this.

Lemma 3.4. If the red and blue subgraphs in every $(\mathcal{F}, \mathcal{G})$-avoidance on $K_{p: r}$ are regular, then every $(\mathcal{F}, \mathcal{G})$-avoidance on $K_{2 p+1: 2 r}$ is constant on consecutive pairs of length classes, each pair consisting of all edges with lengths $2 i$ or $2 i+1$ for some $i$ in $\{r, \ldots,\lfloor p / 2\rfloor\}$.

Proof. The subgraph of $K_{(2 p+1): 2 r}$ induced by $\left\{v_{0}, v_{2}, \ldots, v_{2 p-2}\right\}$ is isomorphic to $K_{p: r}$; call it $K$. The length of the edge from the first to the last of these vertices is 3 instead of 2 . Substituting $v_{2 p}$ for $v_{0}$ yields another copy $K^{\prime}$ of $K_{p: r}$. Given that every $(\mathcal{F}, \mathcal{G})$-avoidance on $K_{p: r}$ is regular, any edge $v_{0} v_{2 i}$ lost from $K$ by deleting $v_{0}$ must be replaced by the edge $v_{2 p} v_{2 i}$ having the same color, for $r \leq i \leq p-r$.

Now fix $i \in\{r, \ldots,\lfloor p / 2\rfloor\}$. Replace $\left\{v_{2 j}: 1 \leq j \leq i-1\right\}$ in $K^{\prime}$ with $\left\{v_{2 j-1}: 1 \leq j \leq i-1\right\}$ to obtain $L_{i}$, and replace $v_{2 i}$ with $v_{2 i-1}$ in $L_{i}$ to form $L_{i}^{\prime}$. By the same argument as before, $v_{2 p} v_{2 i}$ and $v_{2 p} v_{2 i-1}$ have the same color. In particular, $v_{2 p} v_{2 i-1}$ and $v_{0} v_{2 i}$ have the same color. Having shown that consecutive edges of length $2 i$ have the same color, we conclude that all edges of length $2 i$ have the same color. Also, since the consecutive edges were linked by an edge of length $2 i+1$ with that color, the edges of length $2 i+1$ have the same color as the edges of length $2 i$.

Proposition 3.5. $R_{\chi_{c}}\left(K_{4}, K_{4}\right) \leq 17.5$.

Proof. If some vertex in a red/blue edge-coloring of $K_{17}$ has nine neighbors of the same color, then $R\left(K_{3}, K_{4}\right)=9$ yields a monochromatic copy of $K_{4}$. Hence in every $\left(K_{4}, K_{4}\right)$ - 
avoidance on $K_{17: 1}$, every vertex is incident to eight edges of each color. By Lemma 3.4, a $\left(K_{4}, K_{4}\right.$ )-avoidance $f$ on $K_{35: 2}$ must be constant on consecutive pairs of distance classes.

We may assume that the distance pair $\{8,9\}$ is red. Hence the cycle $\left[v_{0}, v_{9}, v_{17}, v_{26}\right]$ is all red. Both chords have length 17 , so the pair $\{16,17\}$ is blue.

If $\{2,3\}$ is red, then $[0,3,5,8] \Rightarrow\{4,5\}$ is blue, $[0,5,17,22] \Rightarrow\{12,13\}$ is red, $[0,2,10,12] \Rightarrow$ $\{10,11\}$ is blue, and $[0,3,9,12] \Rightarrow\{6,7\}$ is blue, leaving $[0,7,17,24]$ as a blue copy of $K_{4}$.

If $\{2,3\}$ is blue, then $[0,2,17,19] \Rightarrow\{14,15\}$ is red, $[0,3,16,19] \Rightarrow\{12,13\}$ is red, $[0,6,14,20] \Rightarrow\{6,7\}$ is blue, $[0,7,17,24] \Rightarrow\{10,11\}$ is red, and $[0,5,15,20] \Rightarrow\{4,5\}$ is blue, leaving $[0,2,4,6]$ as a blue copy of $K_{4}$.

Hence there is no $\left(K_{4}, K_{4}\right)$-avoidance on $K_{35: 2}$.

Question 3.6. What is the value of $R_{\chi_{c}}\left(K_{4}, K_{4}\right)$ ?

Possibly $R_{\chi_{c}}\left(K_{4}, K_{4}\right)=17$, which would be proved by showing $K_{17 q+1: q} \rightarrow K_{4}$ for infinitely many $q$. Each such $q$ yields an upper bound; Proposition 3.5 does this for $q=2$.

Next we further explore colorings in which the color of an edge in $K_{p: q}$ is determined only by its length. This leads to upper and lower bounds on $R_{\chi_{c}}(G)$ for all $G$ with $2<\chi_{c}(G) \leq 3$. From Proposition 3.1, Remark 2.6, and the fact that 4-colorable graphs decompose into two bipartite graphs we already know $4 \leq R_{\chi_{c}}(G) \leq 6$ when $2<\chi_{c}(G) \leq 3$, but now we improve the bounds. They become more accurate when $\chi_{c}(G)$ is close to 3 , and indeed $\lim _{\chi_{c}(G) \rightarrow 3^{-}} R_{\chi_{c}}(G)=6$. In Section 5 we prove $R_{\chi_{c}}(G)=4$ whenever $2<\chi_{c}(G) \leq \frac{5}{2}$.

Lemma 3.7. In $K_{(6 q-1): q}$, the edges of lengths $q$ through $2 q-1$ form a subgraph isomorphic to $K_{(6 q-1): 2 q}$.

Proof. Redraw the graph by putting $v_{i}$ in position $2 i$ (modulo $6 q-1$ ) in the ordering of the vertices around a circle. The edge to $v_{j}$ from $v_{i}$ now has length $2(j-i)$. Thus edges of lengths $q, q+1, \ldots, 2 q-1$ from $v_{i}$ become edges of lengths $2 q, 2 q+2, \ldots, 4 q-2$, while edges of lengths $-q,-q-1, \ldots,-2 q+1$ become edges of lengths $-2 q,-2 q-2, \ldots,-4 q+2$, which equal $4 q-1,4 q-3, \ldots, 2 q+1$. Thus in the redrawing two vertices are adjacent if and only if they are separated by at least $2 q$ positions, as desired.

Since no vertex can have three incident edges of one color, every $\left(K_{3}, K_{3}\right)$-avoidance on $K_{5}$ has complementary monochromatic 5-cycles. Again we take advantage of regularity.

Lemma 3.8. Let $H_{t}=K_{\left(3 \cdot 2^{t}-1\right): 2^{t-1}}$. For $t \geq 2$, the only $\left(K_{3}, K_{3}\right)$-avoidance $f$ on $H_{t}$ gives one color to all edges with length at least $2^{t}$ and the other color to all shorter edges. Each color class is a copy of $K_{\left(3 \cdot 2^{t}-1\right): 2^{t}}$.

Proof. Let $n=3 \cdot 2^{t}-1$. By Lemma 3.7, it suffices to show that $f$ is as described, since the two color classes then both form $K_{n: 2^{t}}$. Since every $\left(K_{3}, K_{3}\right)$-avoidance on $K_{5: 1}$ is regular, by Lemma 3.4 every $\left(K_{3}, K_{3}\right)$-avoidance on $K_{11: 2}$ is constant on pairs of length classes, with 
edges of lengths 2 and 3 having the same color, which edges of length 4 and 5 also have the same color. The two sets must have different colors, completing the description.

Lemma 3.4 now applies iteratively to show that every $\left(K_{3}, K_{3}\right)$-avoidance $f$ on $H_{t}$ is constant on length classes, but we need a stronger statement about the distribution of the classes. The subgraph of $H_{t}$ induced by $\left\{v_{2 i}: 0 \leq i \leq 3 \cdot 2^{t-1}-2\right\}$ is isomorphic to $H_{t-1}$, since consecutive indices are separated by two positions except for one pair separated by three positions. By the induction hypothesis, all edges of length $2^{t-2}$ through $2^{t-1}-1$ in this copy $H$ of $H_{t-1}$ are red, and the longer edges are all blue. Some edges of a given length in $H$ have twice that length in $H_{t}$, and some have twice that length plus 1 . Over all copies of $H_{t-1}$ obtained by rotation, the red edges consist of all edges of lengths $2^{t-1}$ through $2^{t}-1$ in $H_{t}$, and similarly the blue edges consist of all the longer edges.

Theorem 3.9. Let $z_{t}=3-2^{-t}$. If $z_{t-1} \leq \chi_{c}(G) \leq z_{t}$ for $t \geq 1$, then $2 z_{t-1} \leq R_{\chi_{c}}(G) \leq 2 z_{t}$.

Proof. For $t \geq 1$, let $G_{t}=K_{\left(3 \cdot 2^{t}-1\right): 2^{t}}$ and $H_{t}=K_{\left(3 \cdot 2^{t}-1\right): 2^{t-1}}$, so $\chi_{c}\left(G_{t}\right)=z_{t}$ and $\chi_{c}\left(H_{t}\right)=$ $2 z_{t}$. By Lemma 3.8, we have $H_{t} \rightarrow \operatorname{Hom}\left(G_{t}\right)$ and $H_{t-1} \nrightarrow \rightarrow \operatorname{Hom}\left(G_{t}\right)$. By Remark 2.6, this yields $2 z_{t-1} \leq R_{\chi_{c}}\left(G_{t}\right) \leq 2 z_{t}$. Furthermore, since $G_{t}$ is a homomorphic image of every graph with circular chromatic number at most $z_{t}$, and the coloring of $H_{t-1}$ in Lemma 3.8 has no monochromatic subgraph with circular chromatic number bigger than $z_{t-1}$, we have $R_{\chi_{c}}(G) \leq 2 z_{t}$ and $R_{\chi_{c}}(G) \geq 2 z_{t-1}$ whenever $z_{t-1} \leq \chi_{c}(G) \leq z_{t}$.

A slightly different argument about the length classes proves for $q \in\{2,3,4\}$ that $R_{\chi_{c}}(G) \leq 5+\frac{1}{q}$ when $\chi_{c}(G) \leq \frac{5}{2}+\frac{1}{2 q}$. This improves a few bounds.

\section{Larger Values}

Since $K_{p: q} \rightarrow G$ immediately yields $R_{\chi_{c}}\left(G^{\prime}\right) \leq p / q$ for all $G^{\prime}$ such that $\chi_{c}\left(G^{\prime}\right) \leq \chi_{c}(G)$, one naturally wonders how small $R_{\chi_{c}}$ can be among graphs with the same circular chromatic number. Let $R_{\chi_{c}}(z)=\inf \left\{R_{\chi_{c}}(G): \chi_{c}(G) \geq z\right\}$ and $R_{\chi}(k)=\inf \left\{R_{\chi}(G): \chi(G)=k\right\}$ Using the result of Zhu [15] that $R_{\chi}(k)=(k-1)^{2}+1$ (conjectured in [2]), we obtain

$$
\begin{aligned}
R_{\chi_{c}}(k) & =\inf \left\{R_{\chi_{c}}(G): \chi_{c}(G) \geq k\right\} \leq \inf \left\{R_{\chi}(G): \chi_{c}(G) \geq k\right\} \\
& \leq \inf \left\{R_{\chi}(G): \chi(G) \geq k+1\right\}=k^{2}+1 .
\end{aligned}
$$

We use $\chi_{c}(G) \geq z$ instead of $\chi_{c}(G)=z$ in the definition of $R_{\chi_{c}}(z)$ because it is not clear that $\inf \left\{R_{\chi_{c}}(G): \chi_{c}(G)=k\right\} \leq \inf \left\{R_{\chi}(G): \chi(G)=k\right\}$.

We prove the stronger inequality $R_{\chi_{c}}(k) \leq k(k-1)$ using the method of [15]. The fractional chromatic number of a graph $G$, written $\chi_{f}(G)$, is the linear programming relaxation of the chromatic number. That is, $\chi_{f}(G)$ is the minimum sum of weights on the independent sets in $G$ such that each vertex receives total weight at least 1. A $(p, q)$-coloring of $G$ provides such a weighting with total weight $p / q$, so $\chi_{f}(G) \leq \chi_{c}(G)$. 
The categorical or direct product $G \times H$ of graphs $G$ and $H$ has vertex set $V(G) \times V(H)$, with $(u, v)$ and $\left(u^{\prime}, v^{\prime}\right)$ adjacent if and only if $u u^{\prime} \in E(G)$ and $v v^{\prime} \in E(H)$. By coloring $G \times H$ according to a proper coloring $\phi$ of one factor (for example, let the color of $(u, v)$ be $\phi(u)$ for all $v$ ), always $\chi(G \times H) \leq \min \{\chi(G), \chi(H)\}$, and the corresponding inequalities for $\chi_{c}$ and $\chi_{f}$ also hold. Hedetniemi [7] conjectured that always $\chi(G \times H)=\min \{\chi(G), \chi(H)\}$. Zhu [15] proved the equality for $\chi_{f}$, and Tardif [12] proved the equality for $\chi_{c}$ when the minimum over the factors is at most 4 .

Theorem $4.1([15])$. Always $\chi_{f}(G \times H)=\min \left\{\chi_{f}(G), \chi_{f}(H)\right\}$.

Theorem $4.2([12])$. If $\min \left\{\chi_{c}(G), \chi_{c}(H)\right\} \leq 4$, then $\chi_{c}(G \times H)=\min \left\{\chi_{c}(G), \chi_{c}(H)\right\}$.

Iterating the product yields $\chi_{f}\left(G_{1} \times \cdots \times G_{t}\right)=\min \left\{\chi_{f}\left(G_{1}\right), \ldots, \chi_{f}\left(G_{t}\right)\right\}$. Similarly, $\chi_{c}\left(G_{1} \times \cdots \times G_{t}\right)=\min \left\{\chi_{c}\left(G_{1}\right), \ldots, \chi_{c}\left(G_{t}\right)\right\}$ if the minimum is at most 4 .

Lemma 4.3. If every 2-edge-coloring of a graph $H$ contains a monochromatic subgraph with fractional chromatic number at least $z$, then there exists a graph $G$ with $\chi_{f}(G) \geq z$ such that $H \rightarrow \operatorname{Hom}(G)$. When $z \leq 4$, the same statement holds for circular chromatic number.

Proof. Let $t$ be the number of 2-edge-colorings of $H$. Let $G_{i}$ be a graph with fractional chromatic number at least $z$ that occurs as a monochromatic subgraph in the $i$ th coloring. Let $G=G_{1} \times \cdots \times G_{t}$. Each $G_{i}$ is a homomorphic image of $G$, obtained by mapping the independent sets having a fixed value in the $i$ th coordinate into the corresponding vertices in $G_{i}$. Hence $H \rightarrow \operatorname{Hom}(G)$, by construction.

The statement about $\chi_{f}(G)$ or $\chi_{c}(G)$ follows from Theorem 4.1 or Theorem 4.2. In the latter case, if the circular chromatic numbers of the specified monochromatic subgraphs all exceed 4 , then considering a 4 -chromatic subgraph of one of them yields $\chi_{c}(G) \geq z$.

In the next result, the comment about circular chromatic number improves the upper bound on $R_{\chi_{c}}(z)$ when $z \leq 4$, because a monochromatic subgraph with $\chi_{c} \geq z$ may be forced by $K_{p: q}$ with smaller $p / q$ than needed for $\chi_{f} \geq z$, since always $\chi_{c} \geq \chi_{f}$.

Lemma 4.4. If every 2-edge-coloring of $K_{p: q}$ contains a monochromatic subgraph with fractional chromatic number at least $z$, then $R_{\chi_{c}}(z) \leq p / q$; that is, there exists a graph $G$ with $\chi_{c}(G) \geq z$ and $R_{\chi_{c}}(G) \leq p / q$. When $z \leq 4$, the same conclusion follows also when the monochromatic subgraphs are only required to have circular chromatic number at least $z$.

Proof. By Lemma 4.3, there exist a graph $G$ with $\chi_{f}(G) \geq z$ and $K_{p: q} \rightarrow \operatorname{Hom}(G)$. Since $\chi_{c}(G) \geq \chi_{f}(G)$ and $R_{\chi_{c}}(G)=\inf \left\{p / q: K_{p: q} \rightarrow \operatorname{Hom}(G)\right\}$, the claim follows. When $z \leq 4$, applying the second statement of Lemma 4.3 yields $R_{\chi_{c}}(z) \leq p / q$ directly.

Theorem 4.5. $R_{\chi_{c}}(k) \leq k(k-1)$ for $k \in \mathbb{N}-\{1\}$. 
Proof. Let $R$ and $B$ be the spanning subgraphs formed by the color classes in a red/blue edge-coloring of $K_{k(k-1)}$. If $R$ has a clique of size $k$, then $\chi_{f}(R) \geq k$; otherwise, $B$ has independence number at most $k-1$, and then $\chi_{f}(B) \geq k(k-1) /(k-1)=k$. Since $K_{k(k-1)}=K_{k(k-1): 1}$, the claim follows from Lemma 4.4 .

Note that although Theorem 4.5 applies only to integers, it is consistent with the bounds obtained in Theorem 3.9 .

Question 4.6. Is it true for all $z \in \mathbb{R}$ with $z>2$ that $R_{\chi_{c}}(z) \leq z(\lceil z\rceil-1)$ ? If true, is the bound sharp?

\section{Odd Cycles}

When $k=1$, Theorem 3.9 yields $4 \leq R_{\chi_{c}}(G) \leq 5$ when $2 \leq \chi_{c}(G) \leq \frac{5}{2}$. In fact, we will show $R_{\chi_{c}}(G)=4$ for all such graphs. As we have observed in Corollary 2.5, this yields $R_{\chi_{c}}(\mathcal{F}, \mathcal{G})$ whenever $\mathcal{F}$ and $\mathcal{G}$ both consist of nonbipartite subgraphs and have a member with circular chromatic number at most 5.

Our main task is to prove $R_{\chi_{c}}\left(C_{5}\right)=4$. Since $C_{3}$ and $C_{5}$ are homomorphic images of $C_{5}$, we accomplish this by proving for $q \geq 1$ that every 2-edge-coloring of $K_{4 q+1: q}$ has a monochromatic 3-cycle or a monochromatic 5-cycle.

Let $K_{p: q}^{-}$denote $K_{p: q}-v_{0} v_{q}$ (deleting a shortest edge). In this graph we call the endpoints of the edge that was deleted the special pair. Let a 3,5-free coloring of a graph $G$ be a 2-edge-coloring having no monochromatic 3-cycle or 5-cycle.

Lemma 5.1. Every 3,5-free coloring of $K_{5: 1}^{-}$has monochromatic paths of length 2 in both colors joining $v_{0}$ and $v_{4}$, the endpoints of the missing edge.

Proof. Consider a 3,5-free coloring. There are nine edges; let red be the larger class. Each color class must be bipartite.

There are five or six red edges, since the maximum number of edges in a bipartite subgraph of $K_{5}$ is 6 , achieved only by $K_{2,3}$. To have at least five red edges, the partite sets of the red graph must have sizes 2 and 3 . Hence the red graph is $K_{2,3}$ with at most one edge deleted.

Since the blue graph must not contain a triangle, the partite set of size 3 must contain $\left\{v_{0}, v_{4}\right\}$. Now there is a blue path joining them through the third vertex of that part. There is a red path joining them via the other partite set, because there are two such possible paths and at most one edge was deleted from $K_{2,3}$ to form the red graph.

Lemma 5.2. In $V\left(K_{4 q+1: q}^{-}\right)$, let $S=\left\{v_{0}, v_{q}, v_{2 q+1}, v_{3 q+1}\right\}$ and $T=\left\{v_{0}, v_{q}, v_{2 q}, v_{3 q+1}\right\}$. Both $K_{4 q+1: q}^{-}-S$ and $K_{4 q+1: q}^{-}-T$ are isomorphic to $K_{4(q-1)+1: q-1}^{-}$, with $\left\{v_{q+1}, v_{2 q}\right\}$ being the special pair when $S$ is deleted and $\left\{v_{2 q+1}, v_{3 q}\right\}$ being the special pair when $T$ is deleted. 
Proof. The vertices of $S$ or $T$ are spaced by $q, q, q, q+1$ (in cyclic order) along the indexing. Hence when $S$ or $T$ is deleted, any two vertices at least $q-1$ steps apart in the new indexing were separated by a deleted vertex and hence were at least $q$ steps apart in the old indexing, except the pair $\left\{v_{q+1}, v_{2 q}\right\}$ in the first case and the pair $\left\{v_{2 q+1}, v_{3 q}\right\}$ in the second case. Hence the edges are those of $K_{4(q-1)+1: q-1}^{-}$, with the special pairs as specified.

When $q=2$, the special pairs in the two resulting subgraphs in Lemma 5.2 are $\left\{v_{3}, v_{4}\right\}$ and $\left\{v_{5}, v_{6}\right\}$. In the inductive proof of the main theorem, we will combine Lemma 5.2 with the following technical result about these two pairs in $K_{9: 2}^{-}$. Write a path or cycle with vertices $v_{1}, \ldots, v_{n}$ in order as $\left\langle v_{1}, \ldots, v_{n}\right\rangle$ or $\left[v_{1}, \ldots, v_{n}\right]$, respectively.

Lemma 5.3. Any 3,5-free coloring of $K_{9: 2}^{-}-\left\{v_{1}, v_{8}\right\}$ having no monochromatic $v_{3}, v_{4}$-path or $v_{5}, v_{6}$-path of length 3 has monochromatic $v_{0}, v_{2}$-paths of length 2 in both colors.

Proof. Let $G^{\prime}=K_{9: 2}^{-}-\left\{v_{1}, v_{8}\right\}$ and $G=G^{\prime}-v_{0}$, shown in bold in Figure 1. Let $G_{r}$ and $G_{b}$ be the red and blue color classes of $G$ under the given 3,5-free coloring. Since $G$ has only six vertices, $G_{r}$ and $G_{b}$ are bipartite. We prove first that $v_{3}$ and $v_{4}$ are in the same partite set in each of $G_{r}$ and $G_{b}$, as are $v_{5}$ and $v_{6}$. By symmetry, it suffices to forbid $v_{3}$ and $v_{4}$ being in opposite parts in $G_{r}$.

By hypothesis there is no red $v_{3}, v_{4}$-path of length 3 , so being in opposite parts requires a spanning $v_{3}, v_{4}$-path $P$ in $G_{r}$. After $v_{3}$, the next vertex $u$ must be one of $\left\{v_{5}, v_{6}, v_{7}\right\}$. In each case, we obtain a contradiction. If $u=v_{5}$, then $P=\left\langle v_{3}, v_{5}, v_{7}, v_{2}, v_{6}, v_{4}\right\rangle$, but then $\left\langle v_{5}, v_{7}, v_{2}, v_{6}\right\rangle$ is a forbidden red $v_{5}, v_{6}$-path of length 3 . If $u=v_{6}$, then $P=\left\langle v_{3}, v_{6}, v_{2}, v_{5}, v_{7}, v_{4}\right\rangle$. To avoid completing red odd cycles with edges of $P$, both $v_{3} v_{7}$ and $v_{7} v_{2}$ must be blue. Now there are $v_{3}, v_{2}$-paths of length 2 in both colors, and one extends along $v_{2} v_{4}$ to complete a monochromatic $v_{3}, v_{4}$-path of length 3 . If $u=v_{7}$, then $P=\left\langle v_{3}, v_{7}, v_{5}, v_{2}, v_{6}, v_{4}\right\rangle$. To avoid completing red odd cycles with edges of $P$, all of $\left\{v_{7} v_{2}, v_{2} v_{4}, v_{4} v_{7}\right\}$ must be blue, which completes a blue 3-cycle.

Now each of $\left\{v_{3}, v_{4}\right\}$ and $\left\{v_{5}, v_{6}\right\}$ lies in one partite set in both $G_{r}$ and $G_{b}$. Since $\left\{v_{2}, v_{4}, v_{6}\right\}$ and $\left\{v_{3}, v_{5}, v_{7}\right\}$ form triangles, putting all of $v_{3}, v_{4}, v_{5}, v_{6}$ into the same part in $G_{r}$ or $G_{b}$ forces $v_{2}$ and $v_{7}$ into the other part. Similarly, since $\left\{v_{2}, v_{7}\right\}$ cannot lie in the same part with $v_{4}$ or $v_{5}$, putting $\left\{v_{3}, v_{4}\right\}$ and $\left\{v_{5}, v_{6}\right\}$ into opposite parts forces $v_{2}$ and $v_{7}$ into opposite parts. Hence each of the resulting bipartions $R$ and $B$ of the indices has three possibilities: (3456|27), (347|562), and (342|567). Since the edges within a partite set get the other color, each choice for $R$ restricts the choice for $B$. Since the two subgraphs cannot have the same bipartition, by symmetry there remain three cases. In each case we study $G^{\prime}$ to obtain the monochromatic $v_{0}, v_{2}$-paths of length 2 in both colors.

Case 1: $R=(3456 \mid 27), B=(347 \mid 562)$. If $v_{0} v_{7}$ is red, then avoiding $\left[v_{0}, v_{7}, v_{4}\right]$ in red makes $v_{0} v_{4}$ blue. Now avoiding $\left[v_{0}, v_{4}, v_{6}, v_{3}, v_{5}\right]$ in blue makes $v_{0} v_{5}$ red, so $\left\langle v_{0}, v_{5}, v_{2}\right\rangle$ is red. Avoiding $\left[v_{4}, v_{7}, v_{0}, v_{5}, v_{2}\right]$ in red makes $v_{4} v_{2}$ blue, so $\left\langle v_{0}, v_{4}, v_{2}\right\rangle$ is blue. 


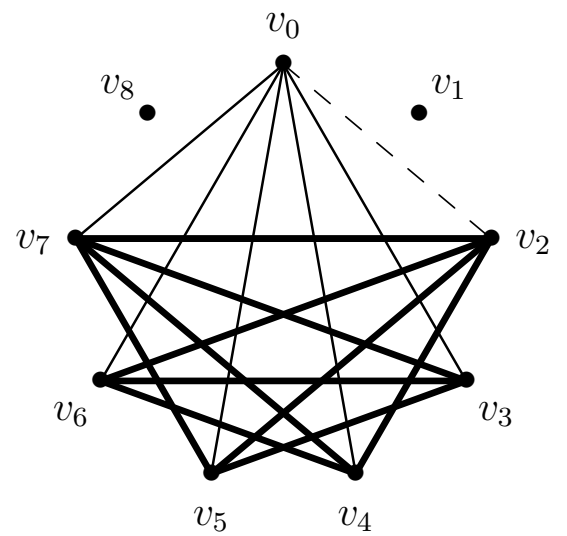

Figure 1: The graphs $G^{\prime}$ and $G$ in Lemma 5.3

If $v_{0} v_{7}$ is blue, then $\left\langle v_{0}, v_{7}, v_{2}\right\rangle$ is blue. Avoiding $\left\langle v_{0}, v_{5}, v_{2}\right\rangle$ and $\left\langle v_{0}, v_{6}, v_{2}\right\rangle$ in red would make $v_{0} v_{5}$ and $v_{0} v_{6}$ blue. Avoiding $\left[v_{0}, v_{4}, v_{6}\right]$ in blue then makes $v_{0} v_{4}$ red. Avoiding $\left[v_{2}, v_{7}, v_{0}, v_{6}, v_{4}\right]$ in blue makes $v_{4} v_{2}$ red, and now $\left\langle v_{0}, v_{4}, v_{2}\right\rangle$ is red.

Case 2: $R=(3456 \mid 27), B=(342 \mid 567)$. If $v_{0} v_{7}$ is red, then avoiding $\left[v_{0}, v_{7}, v_{5}\right]$ in red makes $v_{0} v_{5}$ blue. Now avoiding $\left[v_{0}, v_{5}, v_{3}, v_{6}, v_{4}\right]$ in blue makes $v_{0} v_{4}$ red, and hence $\left\langle v_{0}, v_{4}, v_{2}\right\rangle$ is red. Avoiding $\left[v_{5}, v_{7}, v_{0}, v_{4}, v_{2}\right]$ in red makes $v_{5} v_{2}$ blue, so $\left\langle v_{0}, v_{5}, v_{2}\right\rangle$ is blue.

If $v_{0} v_{7}$ is blue, then $\left\langle v_{0}, v_{7}, v_{2}\right\rangle$ is blue. Avoiding $\left\langle v_{0}, v_{4}, v_{2}\right\rangle$ in red would make $v_{0} v_{4}$ blue, and then avoiding $\left[v_{0}, v_{4}, v_{6}\right]$ in blue makes $v_{0} v_{6}$ red. Avoiding $\left[v_{2}, v_{7}, v_{0}, v_{4}, v_{6}\right]$ in blue makes $v_{2} v_{6}$ red, and now $\left\langle v_{0}, v_{6}, v_{2}\right\rangle$ is red.

Case 3: $R=(342 \mid 567), B=(347 \mid 562)$. If $v_{0} v_{7}$ is red, then avoiding $\left[v_{0}, v_{7}, v_{4}\right]$ in red makes $v_{0} v_{4}$ blue, so $\left\langle v_{0}, v_{4}, v_{2}\right\rangle$ is blue. Now avoiding $\left[v_{0}, v_{4}, v_{2}, v_{7}, v_{5}\right]$ in blue makes $v_{2} v_{7}$ or $v_{0} v_{5}$ red, so $\left\langle v_{0}, v_{7}, v_{2}\right\rangle$ or $\left\langle v_{0}, v_{5}, v_{2}\right\rangle$ is red.

If $v_{0} v_{7}$ is blue, then avoiding $\left[v_{0}, v_{7}, v_{5}\right]$ in blue makes $v_{0} v_{5}$ red, so $\left\langle v_{0}, v_{5}, v_{2}\right\rangle$ is red. Avoiding $\left[v_{0}, v_{5}, v_{2}, v_{7}, v_{4}\right]$ in red makes $v_{2} v_{7}$ or $v_{0} v_{4}$ blue, so $\left\langle v_{0}, v_{7}, v_{2}\right\rangle$ or $\left\langle v_{0}, v_{4}, v_{2}\right\rangle$ is blue.

Theorem 5.4. $R_{\chi_{c}}\left(C_{5}\right)=4$.

Proof. It suffices to show $K_{4 q+1: q} \rightarrow\left\{C_{3}, C_{5}\right\}$ for $q \geq 1$. We use induction on $q$ to prove that every 3,5 -free coloring of $K_{4 q+1: q}^{-}$contains monochromatic $v_{0}, v_{q}$-paths of length 2 in both colors. Adding the edge $v_{0} v_{q}$ then completes a monochromatic triangle. Lemma 5.1 proves the case $q=1$.

For $q>1$, let $G=K_{4 q+1: q}^{-}$, and consider a 3, 5-free coloring of $G$. Let $S=\left\{v_{0}, v_{q}, v_{2 q+1}, v_{3 q+1}\right\}$ and $T=\left\{v_{0}, v_{q}, v_{2 q}, v_{3 q+1}\right\}$. By Lemma 5.2, both $G-S$ and $G-T$ are isomorphic to $K_{4(q-1)+1: q-1}^{-}$, with special pairs $\left\{v_{q+1}, v_{2 q}\right\}$ and $\left\{v_{2 q+1}, v_{3 q}\right\}$, respectively. By the induction hypothesis, there are monochromatic $v_{q+1}, v_{2 q}$-paths and $v_{2 q+1}, v_{3 q}$-paths of length 2 in both colors. A monochromatic $v_{q+1}, v_{2 q}$-path or $v_{2 q+1}, v_{3 q}$-path of length 3 in $G$ would thus complete a monochromatic closed odd walk of length 5 , which would yield a monochromatic 
3 -cycle or 5-cycle, so there is no such path for either pair.

Now consider the subgraph of $G$ induced by $\left\{v_{0}, v_{q}, v_{q+1}, v_{2 q}, v_{2 q+1}, v_{3 q}, v_{3 q+1}\right\}$. This subgraph is isomorphic to $K_{9: 2}^{-}-\left\{v_{1}, v_{8}\right\}$, with the vertices representing $v_{0}, v_{2}, v_{3}, v_{4}, v_{5}, v_{6}, v_{7}$ in order. By Lemma 5.3, there are monochromatic $v_{0}, v_{q}$-paths of length 2 in both colors.

\section{Non-diagonal Ramsey numbers for Cycles}

In this section we prove $4 \leq R_{\chi_{c}}\left(C_{3}, C_{7}\right) \leq \frac{9}{2}<\frac{14}{3} \leq R_{\chi_{c}}\left(C_{3}, C_{5}\right) \leq 5$.

For $R_{\chi_{c}}\left(C_{3}, C_{5}\right)$, we first found a lower bound of $\frac{9}{2}$ using a red/blue coloring of $K_{9: 2}$ that was not constant on distance classes. In fact, this coloring arises by the natural homomorphism from $K_{9: 2}$ into the coloring of $K_{14: 3}$ in Theorem 6.1, which was found by Daniel Cranston). It is not yet known whether distance-invariant optimal lower-bound colorings can always be found for $R_{\chi_{c}}(F, G)$, although certainly such colorings are much easier to study than more general colorings.

Theorem 6.1. $\frac{14}{3} \leq R_{\chi_{c}}\left(C_{3}, C_{5}\right) \leq 5$.

Proof. For the upper bound, it suffices to show $K_{5: 1} \rightarrow\left(\operatorname{Hom}\left(C_{3}\right), \operatorname{Hom}\left(C_{5}\right)\right)$. Note that $\operatorname{Hom}\left(C_{5}\right)=\left\{C_{3}, C_{5}\right\}$. We have remarked that the only red/blue-coloring of $K_{5}$ having no monochromatic triangle has monochromatic 5-cycles in both colors.

For the lower bound, we color $E\left(K_{14: 3}\right)$. Edges of lengths 3 and 4 are blue; edges of lengths 5, 6, and 7 are red. Since the lengths of two red edges sum to at least 10 and the lengths of three sum to at least 15 , there is no red triangle. The lengths of two blue edges sum to at least 6 and the lengths of three sum to at most 12, so there is no blue triangle.

Now consider blue 5-cycles. The cycle may take steps in both directions, but the total net movement in one direction must be a multiple of 14 . With five steps in the same direction, the sum is at least 15 and at most 20. With four edges in one direction (total from 12 to 16) and one in the other (length 3 or 4), the net movement is at least 8 and at most 13, not a multiple of 14. Similarly, three edge in one direction (total from 9 to 12) and two in the other (total from 6 to 8 ) yield net movement at least 1 and at most 6 , again not a multiple of 14 . Hence there is no blue 5-cycle.

Other colorings of $E\left(K_{14: 3}\right)$ also establish this lower bound. One can make the edges of lengths 3 and 4 red, the edges of lengths 6 and 7 blue, and alternate red and blue along the 14-cycle formed by the edges of length 5. Both this and the coloring in Theorem 6.1 have 35 red edges and 28 blue edges, but they are not isomorphic. There is also a messy coloring that is not distance-invariant. This multiplicity of colorings suggests that there could be better colorings, but we show next that they will be hard to find.

Remark 6.2. The construction in Theorem 6.1 does not generalize to give either a larger lower bound for $R_{\chi_{c}}\left(C_{3}, C_{5}\right)$ or a smaller lower bound for $R_{\chi_{c}}\left(C_{3}, C_{2 k+1}\right)$. Consider a red/blue 
coloring of $E\left(K_{p: q}\right)$ with $p / q>4$ such that all edges with lengths from $q$ to $r$ are blue, all edges with lengths from $r+1$ to $\lfloor p / 2\rfloor$ are red, no triangle is red, and no odd cycle of length at most $2 k+1$ is blue. In Theorem 6.1 we have the case $(p, q, r)=(14,3,4)$ and $k=2$.

To avoid red triangles, we must have $r+1>p / 3$. Avoiding blue triangles requires $r<p / 3$. Hence $p \in\{3 r+1,3 r+2\}$. To avoid creating blue 5 -cycles that take three steps forward and two steps back, we need $3 q>2 r$. Now $2 p \leq 6 r+4 \leq 9 q+1$. However, we must also avoid blue 5-cycles with four steps forward and one step back for net movement $p$. The smallest such total is $4 q-r$, the largest is $4 r-q$, and all values between them are achievable. With $q=r-s$, these values range from $3 r-3 s$ to $3 r+s$.

Since $p \in\{3 r+1,3 r+2\}$, the range includes $p$ unless $s=1$ and $p=3 r+2$. Now also $q=r-1$, so $p / q=3+5 / q$. Since we want $4<p / q<5$, the only possible instances are $(p, q, r)=(14,3,4)$ (as in Theorem 6.1) and $(p, q, r)=(17,4,5)$. Since $\frac{17}{4}<\frac{14}{3}$, the second construction would be of interest only for $R_{\chi_{c}}\left(C_{3}, C_{7}\right)$. However, the construction contains a blue 7 -cycle in that case, since $6 \cdot 5+4=2 \cdot 17$.

Making the short edges red and long edges blue also does not succeed.

We next give an upper bound for $R_{\chi_{c}}\left(C_{3}, C_{7}\right)$. Let a good coloring of a graph be a red/blue edge-coloring having no red triangle and no blue triangle or 5-cycle. Recall from Section 5 that the endpoints of the missing edge in $K_{5: 1}^{-}$are $v_{0}$ and $v_{4}$, cyclically indexed.

Lemma 6.3. Good colorings of $K_{5: 1}^{-}$are of two types. One has monochromatic paths of length 2 in both colors joining $v_{0}$ and $v_{4}$. The other consists of a red 5-cycle and a spanning blue path with endpoints $v_{0}$ and $v_{4}$. edge.

Proof. By Lemma 5.1, every good coloring having no monochromatic 3-cycle or 5-cycle is of the first type. Hence we need only consider colorings that have a red 5-cycle. Since $v_{0}$ and $v_{4}$ are not adjacent, they are not consecutive on the cycle. Since $v_{1}, v_{2}$, and $v_{3}$ are in the same isomorphism class, we may assume by symmetry that the cycle visits $v_{0}, v_{1}, v_{4}, v_{2}, v_{3}$ in order. Any red chord creates a red triangle, so the remaining four edges must be blue, yielding the blue path with vertices $v_{0}, v_{2}, v_{1}, v_{3}, v_{4}$ in order.

Theorem 6.4. $R_{\chi_{c}}\left(C_{3}, C_{7}\right) \leq \frac{9}{2}$.

Proof. It suffices to show that every red/blue coloring of $K_{9: 2}$ has a red triangle or a blue odd cycle with length at most 7 . Consider a coloring that avoids this.

If the edges of length 2 are all red, then the edges of length 4 form a blue 9-cycle. Any additional blue edge would be a chord yielding a shorter blue odd cycle, so the edges of length 3 are all red and form triangles.

Hence we may assume by symmetry that the edge $v_{0} v_{2}$ is blue. Consider the copies of $K_{5: 1}^{-}$induced by $\left\{v_{1}, v_{3}, v_{5}, v_{7}, v_{0}\right\}$ and $\left\{v_{2}, v_{4}, v_{6}, v_{8}, v_{1}\right\}$; call them $Q^{-}$and $Q^{+}$, respectively. Note that $Q^{-}$and $Q^{+}$share only $v_{1}$. By Lemma 6.3 , if either $Q^{-}$or $Q^{+}$has a good coloring 
of the first type, then the union of the guaranteed blue $v_{0}, v_{1}$-path in $Q^{-}$, the guaranteed blue $v_{1}, v_{2}$-path in $Q^{+}$, and the edge $v_{2} v_{0}$ is a blue 5 -cycle or 7 -cycle.

If both $Q^{-}$and $Q^{+}$have good colorings of the second type, then the union of the guaranteed blue paths with the edge $v_{2} v_{0}$ is a blue 9-cycle. Again any additional blue edge would yield a shorter blue odd cycle, so the blue graph is precisely a 9-cycle.

If both copies have blue paths of length 4 , then $G$ has a blue 9-cycle and any additional blue edge is a chord and completes a shorter cycle. Therefore, the blue graph is $C_{9}$. Edges joining $V\left(Q^{-}\right)$and $V\left(Q^{+}\right)$must now all be red. These include $v_{0} v_{4}, v_{4} v_{7}, v_{0} v_{6}$, and $v_{6} v_{3}$. To avoid red triangles, $v_{0} v_{7}$ and $v_{0} v_{3}$ must be blue. Now $v_{0}$ has two incident blue edges other than $v_{0} v_{2}$, which contradicts the blue graph being $C_{9}$.

The difficulty of extending the construction of Theorem 6.1 via Remark 6.2 suggests that

perhaps $R_{\chi_{c}}\left(C_{3}, C_{5}\right)=\frac{14}{3}$. The same difficulty, coupled with Theorem 6.4 , suggests that perhaps $R_{\chi_{c}}\left(C_{3}, C_{2 k+1}\right)=4$ for $k \geq 3$.

\section{References}

[1] J. A. Bondy and P. Hell, A note on the star chromatic number. J. Graph Theory 14 (1990), 479-482.

[2] S. Burr, P. Erdős, and L. Lovász, On graphs of Ramsey type, Ars Combinatoria 1 (1976), 167-190.

[3] P. Erdős, R.J. Faudree, C.C. Rousseau, and R.H. Schelp, The size Ramsey number, Period. Math. Hungar. 9 (1978), 145-161.

[4] J. Folkman, Graphs with monochromatic complete subgraphs in every edge coloring. SIAM J. Appl. Math. 18 (1970), 19-24.

[5] R. L. Graham, B. L. Rothchild and J. H. Spencer, Ramsey Theory, John Wiley Es Sons, 1990.

[6] R. E. Greenwood and A. M. Gleason, Combinatorial relations and chromatic graphs, Canad. J. Math. 7 (1955), 1-7.

[7] S. Hedetniemi, Homomorphisms and graph automata, University of Michigan Technical Report 03105-44-T, 1966.

[8] T. Jiang, K.G. Milans, and D.B. West, Degree Ramsey number for cycles and blowups of trees, Europ. J. Combin. 34 (2013), 414-423.

[9] W.B. Kinnersley, K.G. Milans, and D.B. West, Degree Ramsey numbers of graphs, Combin., Probab., Comput. 21 (2012), 229-253. 
[10] J. Nešetřil and V. Rödl, The Ramsey property for graphs with forbidden complete subgraphs. J. Combinatorial Theory Ser. B 20 (1976), 243-249.

[11] V. Rödl and E. Szemerédi, On size Ramsey numbers of graphs with bounded maximum degree, Combinatorica 20 (2000), 257-262.

[12] C. Tardif, Multiplicative graphs and semi-lattice endomorphisms in the category of graphs, J. Combin. Theory Ser. B 45 (2004), 338-345.

[13] A. Vince, Star chromatic number, J. Graph Theory 12 (1988), 551-559.

[14] X. Zhu, Chromatic Ramsey numbers, Discrete Math. 190 (1998), 215-222.

[15] X. Zhu, The fractional version of Hedetniemi's conjecture is true, Europ. J. Combin. 32 (2011), 1168-1175. 